\title{
Soliton Resonances of the Nonisospectral Modified Kadomtsev-Petviashvili Equation
}

\author{
Jiaojiao Yan \\ Zhejiang Institute of Communications, Hangzhou, China \\ E-mail: yanjj@zjvtit.edu.com \\ Received March 24, 2011; revised April 2, 2011; accepted April 5, 2011
}

\begin{abstract}
Many equations possess soliton resonances phenomenon, this paper studies the soliton resonances of the nonisospectral modified Kadomtsev-Petviashvili (mKP) equation by asymptotic analysis.
\end{abstract}

Keywords: Soliton, Resonances, Hirota Bilinear Method, Nonisospectral mKP Equation

\section{Introduction}

In the process of searching for explicit solutions, quite a few systematic methods have been developed, such as inverse scattering transformation [1], Darboux transformations [2], Hirota's bilinear method [3-5], and so on. Among them, the bilinear method first proposed by Hirota provides us with a comprehensive approach to construct exact solutions of nonlinear evolution equations (NEEs). Meanwhile, as the interacting of the solution, soliton resonance has been studied in many papers. Miles obtained resonantly interacting solitary waves of KP equation [6], these solutions are coherent structures that describe the diffraction of a soliton at a corner, and suggest that, under certain conditions, a KP soliton can't turn at a convex corner without separating or otherwise losing its identity. Thus, these structures provide a solution of the problem of "Mach reflection" in water waves, and this phenomenon is now known as soliton resonance. Asymptotic analysis is a very important tool in studying the behaviors of soliton solutions, we call the asymptotic line soliton solutions as $y \rightarrow-\infty$ and as $y \rightarrow-\infty$ the incoming and outgoing line soliton solutions, respectively. The amplitudes, directions and even the number of incoming solitons are in general different from those of the outgoing ones, when resonance occurs two soliton solutions under certain condition resonate and create a new soliton solution.

Multisoliton solutions exhibiting nontrivial spatial structures and interaction patterns were found in many well-known soliton equations. Hirota studied resonances of solitons in one-dimensional space theoretically taking the Sawada-Kotera equation with a nonvanishing boundary condition as an example by his bilinear method [7], in which he pointed out that two solitons at the resonant state fused after colliding with each other, or a soliton splited into two solitons. Other $(1+1)$-dimensional space equations like KdV-SK and Hirota-Satsuma equations [8] and Boussinesq equation [9].

However more emphases are placed on $(2+1)$-dimensional ones, the most relevant with ours like the following: Wadati clarified the fundamental properties of the soliton in KP equation [10], Medina then went further in this equation [11], Pashaev created four virtual soliton resonance solution for KP-II [12], Biondini made use of tau-function in Wronskian to study it [13], after that Isojima studied the parameter regions for resonance and also study the "spider web"-like solution for cKP system $[14,15]$, the approach of the Reference [16] for MKP-II equation allows audiences to interpret the resonance soliton as a composite object of two dissipative solitons in $(1+1)$ dimensions, Hao investigated the resonance of two line solitons of the nonisospectral KP equation [17] which classified the resonance condition clearly. Resonance can also occur in $(3+1)$-dimensional system [18] and even multi-dimensional space [19,20].

In recent years, much attention has been paid to the study of nonisospectral systems [21], as nonisospectral evolution equations are of physical and mathematical importance, which can be used to describe solitary waves in a certain type of non-uniform media with a relaxation effect. The aim of this paper is to clarify the fundamental properties of the soliton resonances in the $(2+1)$-dimensional nonisospectral mKP equation 


$$
\begin{aligned}
& 4 u_{t}+y\left(u_{x x x}-6 u^{2} u_{x}+6 u_{x} \partial^{-1} u_{y}+3 \partial^{-1} u_{y y}\right) \\
& +2 x u_{y}-u^{2}+3 \partial^{-1} u_{y}=0
\end{aligned}
$$

whose Wronskian and Grammian type solutions have been studied by Deng [22] and Zhang [23] respectively.

This letter is organized as follows: in Section 2, the 2and 3-soliton solution of Equation (1.1) will be presented using Hirota's bilinear method. Then 2- and 3-soliton resonances will be studied in Sections 3 and 4 respectively. Finally, concluding remarks are given in Section 5.

\section{2- and 3-Soliton Solutions of the Nonisospectral mKP Equation}

Through the transformation $u=\left(\log \frac{g}{f}\right)_{x}$, Equation (1.1) can be transformed into the bilinear form

$$
\begin{gathered}
D_{y} g \cdot f-D^{2}{ }_{x} g \cdot f=0 \\
4 D_{t} g \cdot f+y\left(D^{3}{ }_{x} g \cdot f+3 D_{x} D_{y} g \cdot f\right) \\
+2 x D_{y} g \cdot f+g_{x} f+g f_{x}=0
\end{gathered}
$$

where $D$ is the well-known Hirota bilinear operator

$$
\begin{aligned}
& D_{x}^{l} D^{m}{ }_{y} D^{n}{ }_{t} a \cdot b \\
&=\left(\partial x-\partial x^{\prime}\right)^{l}\left(\partial y-\partial y^{\prime}\right)^{m}\left(\partial t-\partial t^{\prime}\right)^{n} \\
& \cdot a(x, y, t) b\left(x^{\prime}, y^{\prime}, t^{\prime}\right) \mid x^{\prime} \\
&= x, y^{\prime}=y, t^{\prime}=t
\end{aligned}
$$

If we note the $\mathrm{N}$-soliton solution as $u_{N} \Delta\left(\log \frac{g_{N}}{f_{N}}\right)_{X}$ and

$$
\begin{gathered}
g_{N}=\sum_{\varepsilon=0,1} \exp \left[\sum_{j=1}^{N} \varepsilon_{j}\left(\theta_{j}+\log b_{j}\right)+\sum_{1 \leq j<l} \varepsilon_{j} \varepsilon_{l} A_{j l}\right], \\
f_{N}=\sum_{\varepsilon=0,1} \exp \left[\sum_{j=1}^{N} \varepsilon_{j}\left(\theta_{j}+\log a_{j}\right)+\sum_{1 \leq j<l} \varepsilon_{j} \varepsilon_{l} A_{j l}\right],
\end{gathered}
$$

where the sum is taken over all possible combinations of $\varepsilon_{j}=(0,1)(j=1,2, \cdots, N)$, then the first three soliton solutions are

$$
\begin{gathered}
g_{1}=1+a_{1} \mathrm{e}^{\theta_{1}}, f_{1}=1+b_{1} \mathrm{e}^{\theta_{1}}, \\
g_{2}=1+a_{1} \mathrm{e}^{\theta_{1}}+a_{2} \mathrm{e}^{\theta_{2}}+a_{1} a_{2} \mathrm{e}^{\theta_{1} \theta_{2}-\Delta_{12}}, \\
f_{2}=1+b_{1} \mathrm{e}^{\theta_{1}}+b_{2} \mathrm{e}^{\theta_{2}}+b_{1} b_{2} \mathrm{e}^{\theta_{1} \theta_{2}-\Delta_{12}},
\end{gathered}
$$

$$
\begin{aligned}
g_{3}=1 & +a_{1} \mathrm{e}^{\theta_{1}}+a_{2} \mathrm{e}^{\theta_{2}}+a_{3} \mathrm{e}^{\theta 3}+a_{1} a_{2} \mathrm{e}^{\theta_{1} \theta_{2}-\Delta_{12}}+a_{1} a_{3} \mathrm{e}^{\theta_{1} \theta_{3}-\Delta_{13}} \\
& +a_{2} a_{3} \mathrm{e}^{\theta_{2} \theta_{3}-\Delta_{23}}+a_{1} a_{2} a_{3} \mathrm{e}^{\theta_{1}+\theta_{2}+\theta_{3}-\Delta_{12}-\Delta_{13}-\Delta_{23}}, \\
f_{3}= & +b_{1} \mathrm{e}^{\theta_{1}}+b_{2} \mathrm{e}^{\theta_{2}}+b_{3} \mathrm{e}^{\theta_{3}}+b_{1} b_{2} \mathrm{e}^{\theta_{1} \theta_{2}-\Delta_{12}}+b_{1} b_{3} \mathrm{e}^{\theta_{1} \theta_{3}-\Delta_{13}}, \\
& +b_{2} b_{3} \mathrm{e}^{\theta_{2} \theta_{3}-\Delta_{23}}+b_{1} b_{2} b_{3} \mathrm{e}^{\theta_{1}+\theta_{2}+\theta_{3}-\Delta_{12}-\Delta_{13}-\Delta_{23}},
\end{aligned}
$$

where

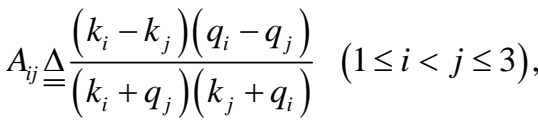

$$
\begin{aligned}
& \theta_{i}=\left(k_{i}+q_{i}\right) x-\left(k_{i}^{2}-q_{i}^{2}\right) y+\delta_{i}, \mathrm{e}_{i}^{\delta}=\omega_{i},
\end{aligned}
$$

$\mathrm{e}^{-\Delta_{i j}}=A_{i j}>0, k_{i}, q_{i}, a_{i}, b_{i}$ and $\omega_{i}$ are all functions corresponding to $t$, which satisfy the following dispersion relations:

$$
\begin{aligned}
& k_{i, t}=\frac{1}{2} k_{i}^{2}, q_{i, t}=-\frac{1}{2} q_{i}^{2}, a_{i}=q_{i}, b_{i}=-k_{i}, \\
& \omega_{i, t}=\frac{1}{4}\left(q_{i}-k_{i}\right) \omega_{i}, \quad(i=1,2,3) .
\end{aligned}
$$

What's more, in order to avoid the divergence of $u$, we suppose $f_{i}$ and $g_{i}$ are all positive. Let $k_{i}+q_{i}=\mu_{i}$ and $k_{i}-q_{i}=v_{i}$, then $\theta_{i}$ can be rewritten as $\theta_{i}=\mu_{i}\left(x-v_{i} y\right)+\delta_{i}$ and without lose of generality we suppose $v_{i}>v_{j}(i>j)$.

\section{2-Solitons}

In general, a soliton is observed when the following two conditions are satisfied:

1) Two terms of Equation (2.3b) are so large that other two terms are neglected.

2) Under the condition 1), the large two terms are of the same order. Under these two conditions, the peak of the soliton is on the line $\theta_{i}=$ cons $\tan t$.

\subsection{Pure 2-Soliton}

When $0<A_{i}<\infty$ and $\neq 1$, for the limit $y \rightarrow-\infty$, $\theta_{1}>\theta_{2}$ the condition 1) and 2) are satisfied in two regions:

$$
\begin{cases}u^{(1)}=\left(\log \frac{1+a_{1} \mathrm{e}^{\theta_{1}}}{1+b_{1} \mathrm{e}^{\theta_{1}}}\right) x, & \theta_{1} \approx 0, \theta_{2} \rightarrow-\infty \\ u^{(2)}=\left(\log \frac{a_{1} \mathrm{e}^{\theta_{1}}+a_{1} a_{2} \mathrm{e}^{\theta_{1} \theta_{2}-\Delta_{12}}}{b_{1} \mathrm{e}^{\theta_{1}}+b_{1} b_{2} \mathrm{e}^{\theta_{1} \theta_{2}-\Delta_{12}}}\right) x, & \theta_{1} \rightarrow+\infty, \theta_{2} \approx \Delta_{12}\end{cases}
$$

so when $y \rightarrow-\infty, u=u^{(1)}+u^{(2)}$. As

$$
\left(\log \frac{a_{1} \mathrm{e}^{\theta_{1}}+a_{1} a_{2} \mathrm{e}^{\theta_{1} \theta_{2}-\Delta_{12}}}{b_{1} \mathrm{e}^{\theta_{1}}+b_{1} b_{2} \mathrm{e}^{\theta_{1} \theta_{2}-\Delta_{12}}}\right)_{x}=\left(\log \frac{1+a_{2} \mathrm{e}^{\theta_{2}-\Delta_{12}}}{1+b_{2} \mathrm{e}^{\theta_{2}-\Delta_{12}}}\right)_{x},
$$


we will use the simplified for later convenience.

Similarly, when, $y \rightarrow+\infty$,

$u=\left(\log \frac{1+a_{1} \mathrm{e}^{\theta_{1}-\Delta_{12}}}{1+b_{1} \mathrm{e}^{\theta_{1}-\Delta_{12}}}\right)_{x}+\left(\log \frac{1+a_{2} \mathrm{e}^{\theta_{2}}}{1+b_{2} \mathrm{e}^{\theta_{2}}}\right)_{x}$ Above all, both of them have four arms and displays the regular interaction, that means two soliton solutions maintain their original amplitudes and velocities during the interaction (See Figure 1).

\subsection{Soliton Resonances}

When $A_{12} \rightarrow+0$, or $A_{12} \rightarrow+\infty$, the phase shift $\left|\Delta_{12}\right|$, becomes $+\infty$, the length of the intermediate region becomes infinite, this may be thought as "soliton resonance”, and the dispersion relation plays a major role in producing the soliton resonance. Further more, as

$$
\left\{\begin{array}{l}
A_{12} \rightarrow+0 \Leftrightarrow\left(k_{1}-k_{2}\right)\left(q_{1}-q_{2}\right) \rightarrow+0 \\
A_{12} \rightarrow+\infty \Leftrightarrow\left(k_{1}+q_{2}\right)\left(k_{2}+q_{1}\right) \rightarrow+0
\end{array},\right.
$$

we call them as "minus resonance" and "plus resonance” respectively.

\subsubsection{Minus Resonance}

Case 1. By taking $A_{12} \rightarrow+0$, Equation (2.3b) becomes $g_{2}=1+a_{1} \mathrm{e}^{\theta_{1}}+a_{2} \mathrm{e}^{\theta_{2}}, f_{2}=1+b_{1} \mathrm{e}^{\theta_{1}}+b_{2} \mathrm{e}^{\theta_{2}}$, from which we have the asymptotic forms (see Equation (3.3)).

The solution has three arms each of which are exact 1-solitons.

\section{Case 2. Substituting}

$$
\begin{aligned}
& g_{2} \rightarrow A_{12}{ }^{-1} g_{2}, f_{2} \rightarrow A_{12}{ }^{-1} f_{2}, \\
& \mathrm{e}^{\theta_{1}} \rightarrow A_{12}{ }^{-1} \mathrm{e}^{\theta_{1}}, \mathrm{e}^{\theta_{2}} \rightarrow A_{12}{ }^{-1} \mathrm{e}^{\theta_{2}}
\end{aligned}
$$

into Equation (2.4b), get

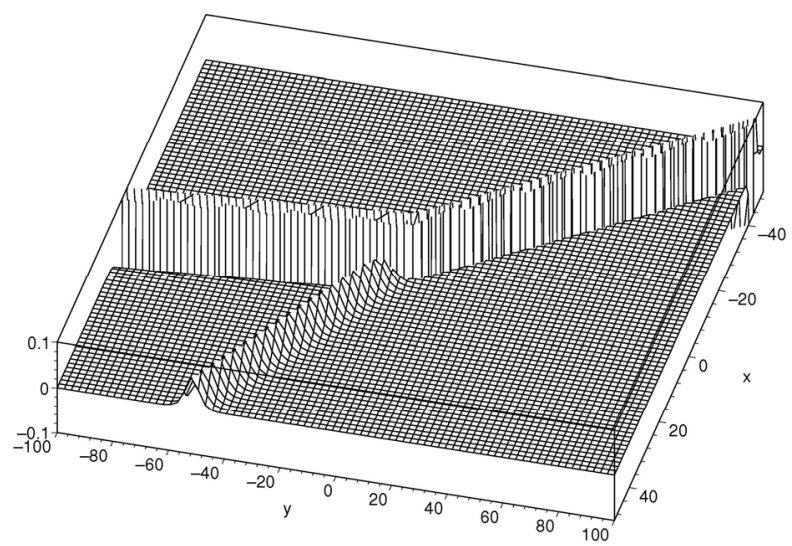

Figure 1. Pure 2-soliton solution.

$$
A_{12} g_{2}=1+a_{1} A_{12}{ }^{-1} \mathrm{e}^{\theta_{1}}+a_{2} A_{12}{ }^{-1} \mathrm{e}^{\theta_{2}}+a_{1} a_{2} A_{12}{ }^{-1} \mathrm{e}^{\theta_{1}+\theta_{2}}
$$

by taking $A_{12} \rightarrow+0$, Equation (3.5) becomes

$$
g_{2}=a_{1} \mathrm{e}^{\theta_{1}}+a_{2} \mathrm{e}^{\theta_{2}}+a_{1} a_{2} \mathrm{e}^{\theta_{1}+\theta_{2}}
$$

Similarly

$$
f_{2}=b_{1} \mathrm{e}^{\theta_{1}}+b_{2} \mathrm{e}^{\theta_{2}}+b_{1} b_{2} \mathrm{e}^{\theta_{1}+\theta_{2}}
$$

The above substitutions are nothing but only a translation of the coordinates.

The corresponding asymptotic forms are Equation (3.7).

The solution has three arms again.

\subsubsection{Plus Resonance}

Case 1. Substituting $\mathrm{e}^{\theta_{1}} \rightarrow A_{12}{ }^{-1} \mathrm{e}^{\theta_{1}}$ into Equation (2.3 b), then taking $A_{12} \rightarrow+\infty$ we get

$$
\begin{aligned}
& g_{2}=1+a_{2} \mathrm{e}^{\theta_{2}}+a_{1} a_{2} \mathrm{e}^{\theta_{1}+\theta_{2}}, \\
& f_{2}=1+b_{2} \mathrm{e}^{\theta_{2}}+b_{1} b_{2} \mathrm{e}^{\theta_{1}+\theta_{2}}
\end{aligned}
$$

$$
\begin{aligned}
& u=\left\{\begin{array}{ll}
u^{(1)}=\left(\log \frac{1+a_{1} \mathrm{e}^{\theta_{1}}}{1+b_{1} \mathrm{e}^{\theta_{1}}}\right)_{x}, & y \rightarrow-\infty, \theta_{1} \approx 0, \theta_{2} \rightarrow-\infty \\
u^{(2)}=\left(\log \frac{1+a_{2} \mathrm{e}^{\theta_{2}}}{1+b_{1} \mathrm{e}^{\theta_{1}}}\right)_{x}, & y \rightarrow+\infty, \theta_{1} \rightarrow-\infty, \theta_{2} \approx 0 \\
u^{(1-2)}=\left(\log \frac{a_{1} \mathrm{e}^{\theta_{1}}+a_{2} \mathrm{e}^{\theta_{2}}}{b_{1} \mathrm{e}^{\theta_{1}}+a_{2} \mathrm{e}^{\theta_{2}}}\right)_{x}, & y \rightarrow+\infty, \theta_{1} \rightarrow+\infty, \theta_{2} \rightarrow+\infty
\end{array} .\right. \\
& u=\left\{\begin{array}{ll}
u^{(2)}=\left(\log \frac{1+a_{2} \mathrm{e}^{\theta_{2}}}{1+b_{2} \mathrm{e}^{\theta_{2}}}\right)_{x}, & y \rightarrow-\infty, \theta_{1} \rightarrow+\infty, \theta_{2} \approx 0 \\
u^{(1)}=\left(\log \frac{1+a_{1} \mathrm{e}^{\theta_{2}}}{1+b_{1} \mathrm{e}^{\theta_{1}}}\right)_{x}, & y \rightarrow+\infty, \theta_{1} \approx \Delta_{12}, \theta_{2} \rightarrow+\infty . \\
u^{(1-2)}=\left(\log \frac{a_{1} \mathrm{e}^{\theta_{1}}+a_{2} \mathrm{e}^{\theta_{2}}}{b_{1} \mathrm{e}^{\theta_{1}}+a_{2} \mathrm{e}^{\theta_{2}}}\right)_{x}, & y \rightarrow-\infty, \theta_{1} \rightarrow-\infty, \theta_{2} \rightarrow+\infty
\end{array} .\right.
\end{aligned}
$$




$$
u=\left\{\begin{array}{lc}
u^{(1+2)}=\left(\log \frac{1+a_{1} a_{2} \mathrm{e}^{\theta_{1}+\theta_{2}}}{1+b_{1} b_{2} \mathrm{e}^{\theta_{1}+\theta_{2}}}\right)_{x}, & y \rightarrow-\infty, \theta_{1}+\theta_{2} \approx 0, \theta_{2} \rightarrow-\infty \\
u^{(1)}+u^{(2)}=\left(\log \frac{1+a_{1} \mathrm{e}^{\theta_{1}}}{1+b_{1} \mathrm{e}^{\theta_{1}}}\right)_{x}+\left(\log \frac{1+a_{2} \mathrm{e}^{\theta_{2}}}{1+b_{2} \mathrm{e}^{\theta_{2}}}\right)_{x}, & y \rightarrow+\infty, \theta_{1} \approx 0, \theta_{2} \rightarrow+\infty \\
\theta_{1} \rightarrow-\infty, \theta_{2} \approx 0
\end{array}\right.
$$

Case 2. Substituting $\mathrm{e}^{\theta_{2}} \rightarrow A_{12}{ }^{-1} \mathrm{e}^{\theta_{2}}$ into (2.3b), then taking $A_{12} \rightarrow+\infty$ we get

$$
\begin{gathered}
g_{2}=1+a_{1} \mathrm{e}^{\theta_{1}}+a_{1} a_{2} \mathrm{e}^{\theta_{1}+\theta_{2}}, f_{2}=1+b_{1} \mathrm{e}^{\theta_{1}}+b_{1} b_{2} \mathrm{e}^{\theta_{1}+\theta_{2}} \\
u=\left\{\begin{array}{lc}
u^{(1)}+u^{(2)}=\left(\log \frac{1+a_{1} \mathrm{e}^{\theta_{1}}}{1+b_{1} \mathrm{e}^{\theta_{1}}}\right)_{x}+\left(\log \frac{1+a_{2} \mathrm{e}^{\theta_{2}}}{1+b_{2} \mathrm{e}^{\theta_{2}}}\right)_{x}, & y \rightarrow-\infty, \theta_{1} \approx 0, \theta_{2} \rightarrow-\infty \\
u^{(1+2)}=\left(\log \frac{1+a_{1} a_{2} \mathrm{e}^{\theta_{1}+\theta_{2}}}{1+b_{1} b_{2} \mathrm{e}^{\theta_{1}+\theta_{2}}}\right)_{x},-\infty, \theta_{2} \approx 0
\end{array}\right. \\
\begin{array}{ll}
\theta_{1} \rightarrow+\infty, \theta_{1} \rightarrow-\infty, \theta_{1}+\theta_{2} \approx 0
\end{array}
\end{gathered}
$$

The above asymptotic analysis discusses the 2-soliton solution and it's two type of resonances, minus resonance and plus resonance, by which we know that they all possess three arms, this theory can be illustrated by Figure 2, and furthermore, they show that when resonance occurs, interaction of two high and steep waves can produce a new weak one.

We have assumed that $v_{1}>v_{2}$, the case of $v_{2}>v_{1}$ is similar, however it is different in the case of $v_{1}=v_{2}$. Let $x-v_{1} y \stackrel{\Delta Z}{=}$, then

$$
\begin{aligned}
& \theta_{1}=\mu_{1} Z+\delta_{1}, \theta_{2}=\mu_{2} Z+\delta_{2}, \\
& A_{12}=\frac{\left(\mu_{1}-\mu_{2}\right)^{2}}{\left(\mu_{1}+\mu_{2}\right)^{2}}
\end{aligned}
$$

where two soliton lie in parallel, this solution is similar to 2-soliton solution of the KdV equation.

\section{3-Solitons}

In this section, we analyze the behaviors in asymptotic regions about typical four types of solutions.

When $0<A_{12}, A_{13}, A_{23}<\infty$ and $\neq 1$, for the limit $y \rightarrow-\infty, \theta_{1}>\theta_{2}>\theta_{3}$, the condition 1) and 2) are satisfied in three regions:

$$
\left\{\begin{aligned}
u^{(1)}= & \left(\log \frac{1+a_{1} \mathrm{e}^{\theta_{1}}}{1+b_{1} \mathrm{e}^{\theta 1}}\right)_{x}, \\
& \theta_{1} \approx 0, \theta_{2} \rightarrow-\infty, \theta_{3} \rightarrow-\infty \\
u^{(2)}= & \left(\log \frac{1+a_{2} \mathrm{e}^{\theta_{2}-\Delta_{12}}}{1+b_{2} \mathrm{e}^{\theta_{2}-\Delta_{12}}}\right)_{x}, \\
& \theta_{1} \rightarrow+\infty, \theta_{2} \approx \Delta_{12}, \theta_{3} \rightarrow+\infty \\
u^{(3)}= & \left(\log \frac{1+a_{3} \mathrm{e}^{\theta_{3}}-\Delta_{13}-\Delta_{23}}{1+b_{3} \mathrm{e}^{\theta_{3}}-\Delta_{13}-\Delta_{23}}\right)_{x}, \\
& \theta_{1} \rightarrow+\infty, \theta_{2} \rightarrow+\infty, \theta_{3} \approx \Delta_{13}
\end{aligned}\right.
$$

so when $y \rightarrow-\infty, u=u^{(1)}+u^{(2)}+u^{(3)}$.

Similarly, when $y \rightarrow+\infty$

$$
\begin{aligned}
u= & \left(\log \frac{1+a_{1} \mathrm{e}^{\theta_{1}}-\Delta_{13}-\Delta_{12}}{1+b_{1} \mathrm{e}^{\theta_{1}}-\Delta_{13}-\Delta_{12}}\right)_{x} \\
& +\left(\log \frac{1+a_{2} \mathrm{e}^{\theta_{2}}-\Delta_{12}}{1+b_{2} \mathrm{e}^{\theta_{2}}-\Delta_{12}}\right)_{x} \\
& +\left(\log \frac{1+a_{3} \mathrm{e}^{\theta_{3}}}{1+a_{3} \mathrm{e}^{\theta_{3}}}\right)_{x} .
\end{aligned}
$$

The above limit analysis can prove that 3-soliton solution has 6 arms on theory, Figure 3 can illustrate it too.

The soliton resonance occurs when one or two or even three of $\Delta_{i j} \rightarrow \pm \infty$, we call them 1-, 2-, 3-resonance solution respectively, each of which include minus resonance and plus resonance, in the following, we will discuss them all.

\subsection{1-Resonance}

In this case, one of $\Delta_{i j} \rightarrow \pm \infty$, we suppose $\Delta_{13} \rightarrow \pm \infty$ without lose of generality, that is equal to $A_{13} \rightarrow \pm 0$ (minus 1-resonance) and $A_{13} \rightarrow \pm \infty$ (plus 1-resonance).

\subsubsection{Minus 1-Resonance}

Taking the limit of $A_{13} \rightarrow \pm 0$, Equation (2.3c) becomes

$$
\begin{aligned}
g_{3}= & 1+a_{1} \mathrm{e}^{\theta_{1}}+a_{2} \mathrm{e}^{\theta_{2}}+a_{3} \mathrm{e}^{\theta_{31}} \\
& +a_{1} a_{2} \mathrm{e}^{\theta_{1}+\theta_{2}-\Delta_{12}}+a_{2} a_{3} \mathrm{e}^{\theta_{2}+\theta_{3}-\Delta_{23}} \\
f_{2}= & 1+b_{1} \mathrm{e}^{\theta_{1}}+b_{2} \mathrm{e}^{\theta_{2}}+b_{3} \mathrm{e}^{\theta_{2}} \\
& +b_{1} b_{3} \mathrm{e}^{\theta_{1}+\theta_{2}-\Delta_{12}}+b_{2} b_{3} \mathrm{e}^{\theta_{2}+\theta_{3}-\Delta_{23}}
\end{aligned},
$$

consequently, the asymptotic forms of the solution are given by 


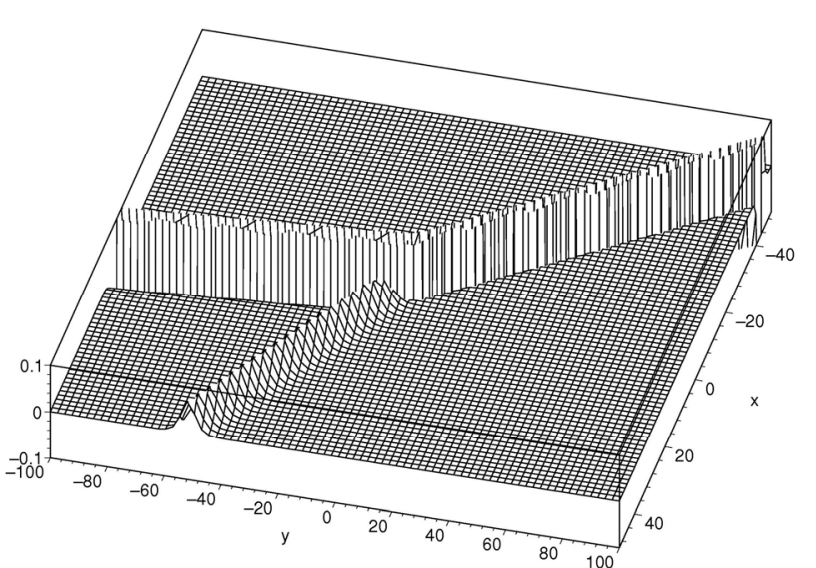

(a)

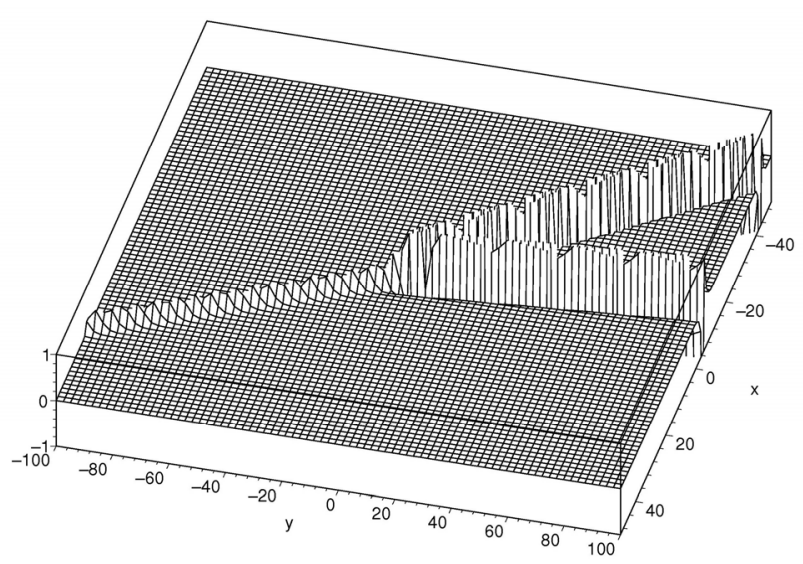

(c)

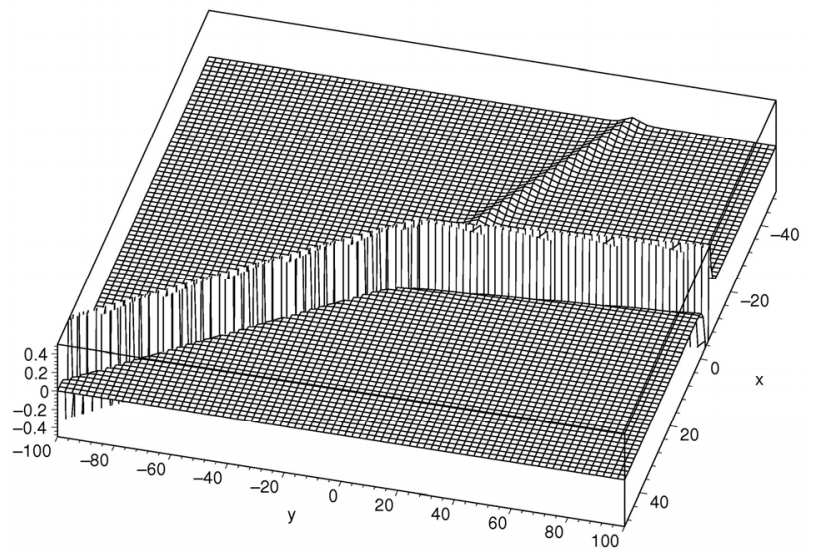

(b)

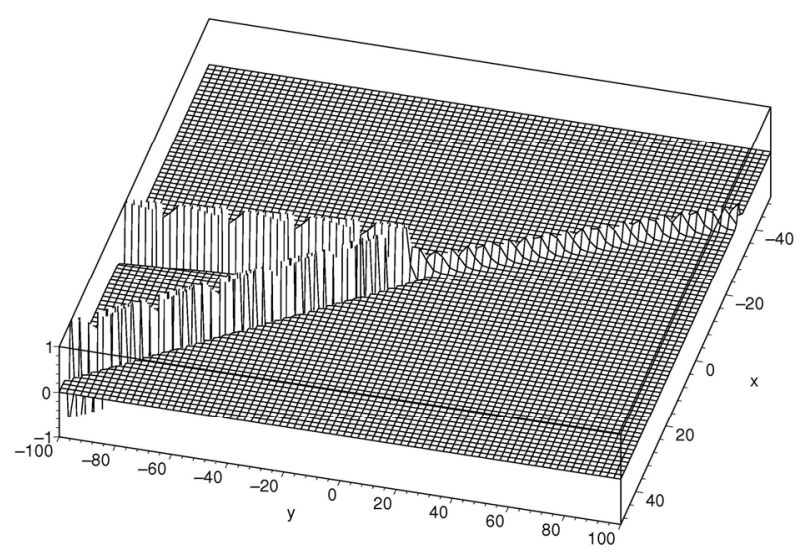

(d)

Figure 2. Minus and plus resonance of 2-soliton solution, (a) 2-soliton minus 1; (b) 2-soliton minus case 2; (c) 2-soliton plus 1; (d) 2-soliton plus case 2 .

$$
u=\left\{\begin{array}{c}
u^{(1)}+u^{(2)}=\left(\log \frac{1+a_{1} \mathrm{e}^{\theta_{1}}}{1+b_{1} \mathrm{e}^{\theta_{1}}}\right)_{x}+\left(\log \frac{1+a_{2} \mathrm{e}^{\theta_{2}-\Delta_{12}}}{1+b_{2} \mathrm{e}^{\theta_{2}-\Delta_{12}}}\right)_{x}, \\
y \rightarrow-\infty \\
u^{(2)}+u^{(3)}=\left(\log \frac{1+a_{2} \mathrm{e}^{\theta_{2}-\Delta_{23}}}{1+b_{2} \mathrm{e}^{\theta_{2}-\Delta_{23}}}\right)_{x}+\left(\log \frac{1+a_{3} \mathrm{e}^{\theta_{1}}}{1+b_{1} \mathrm{e}^{\theta_{1}}}\right)_{x}, \\
y \rightarrow+\infty \\
u^{(1-3)}=\left(\log \frac{a_{1} \mathrm{e}^{\theta_{1}-\Delta_{12}}+a_{3} \mathrm{e}^{\theta_{3}-\Delta_{23}}}{b_{1} \mathrm{e}^{\theta_{1}-\Delta_{12}}+b_{3} \mathrm{e}^{\theta_{3}-\Delta_{23}}}\right)_{x}, x \rightarrow+\infty
\end{array}\right.
$$$$
u=u^{(2)}=\left(\log \frac{1+a_{2} \mathrm{e}^{\theta_{2}-\Delta_{12}-\Delta_{23}}}{1+b_{2} \mathrm{e}^{\theta_{2}-\Delta_{12}-\Delta_{23}}}\right)_{x} .
$$

It is clearly that plus 1-resonance of 2-soliton solution

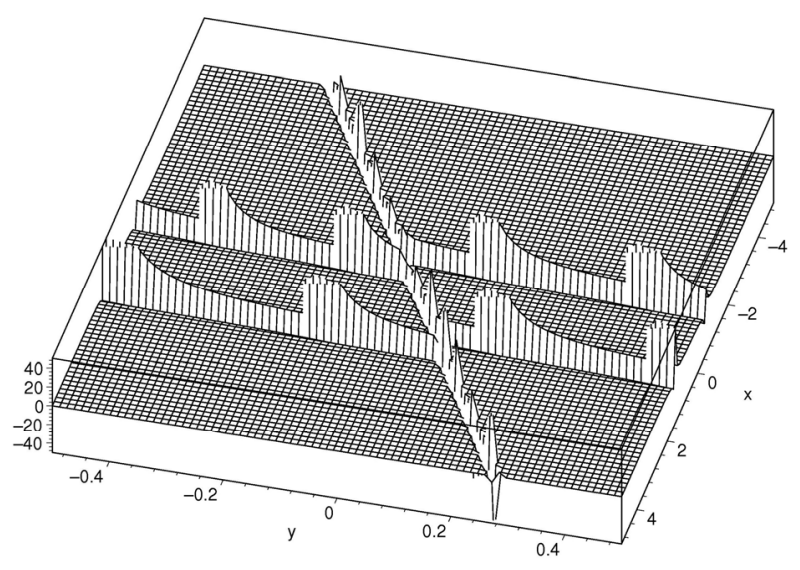

Figure 3. Pure 3-soliton solution. 
only has one arm, and the figure is similar to that of 1-solition solution.

\subsection{2-Resonance}

In this case, two of $\Delta_{i j} \rightarrow \pm \infty$, we suppose $\Delta_{12} \rightarrow \pm \infty$, $\Delta_{23} \rightarrow \pm \infty$ without lose of generality, which are equal to $\Delta_{12} \rightarrow+0, \Delta_{23} \rightarrow+0$ (minus 2-resonance) and $\Delta_{12} \rightarrow+\infty, \Delta_{23} \rightarrow+\infty$ (plus 2-resonance).

\subsubsection{Plus 2-Resonance}

Case 1. Substituting $A_{12} \mathrm{e}^{\theta_{2}} \rightarrow \mathrm{e}^{\theta_{2}}, A_{23} \mathrm{e}^{\theta_{3}} \rightarrow \mathrm{e}^{\theta_{3}}$ into Equation (2.3c), and taking the limit of $A_{12}$ and $A_{13}$, we get

$$
\begin{aligned}
& g_{3}=1+a_{1} \mathrm{e}^{\theta_{1}}+a_{1} a_{2} \mathrm{e}^{\theta_{1}+\theta_{2}}+a_{1} a_{2} a_{3} \mathrm{e}^{\theta_{1}+\theta_{2}+\theta_{3}-\Delta_{13}} \\
& f_{3}=1+b_{1} \mathrm{e}^{\theta_{1}}+b_{1} b_{2} \mathrm{e}^{\theta_{1}+\theta_{2}}+b_{1} b_{2} b_{3} \mathrm{e}^{\theta_{1}+\theta_{2}+\theta_{3}-\Delta_{13}}
\end{aligned}
$$

Then

$$
u=\left\{\begin{array}{l}
u^{(3)}+u^{(2)}+u^{(1)}=\left(\log \frac{1+a_{3} \mathrm{e}^{\theta_{3}-\Delta_{13}}}{1+b_{3} \mathrm{e}^{\theta_{3}-\Delta_{13}}}\right)_{x} \\
\quad\left(\log \frac{1+a_{2} \mathrm{e}^{\theta_{2}}}{1+b_{2} \mathrm{e}^{\theta_{2}}}\right)_{x}+\left(\log \frac{1+a_{1} \mathrm{e}^{\theta_{1}}}{1+b_{1} \mathrm{e}^{\theta_{1}}}\right)_{x}, y \rightarrow-\infty, \\
u^{(1+2+3)}=\left(\log \frac{1+a_{1} a_{2} a_{3} \mathrm{e}^{\theta_{1}+\theta_{2}+\theta_{3}-\Delta_{13}}}{1+b_{1} b_{2} b_{3} \mathrm{e}^{\theta_{1}+\theta_{2}+\theta_{3}-\Delta_{13}}}\right)_{x}, y \rightarrow+\infty .
\end{array}\right.
$$

Case 2. Substituting $A_{12} \mathrm{e}^{\theta_{1}} \rightarrow \mathrm{e}^{\theta_{1}}, A_{23} \mathrm{e}^{\theta_{2}} \rightarrow \mathrm{e}^{\theta_{2}}$ into Equation (2.3c), and taking the limit of $A_{12}$ and $A_{23}$, we get

$$
\begin{aligned}
& g_{3}=1+a_{3} \mathrm{e}^{\theta_{3}}+a_{2} a_{3} \mathrm{e}^{\theta_{2}+\theta_{3}}+a_{1} a_{2} a_{3} \mathrm{e}^{\theta_{1}+\theta_{2}+\theta_{3}-\Delta_{13}} \\
& f_{3}=1+b_{3} \mathrm{e}^{\theta_{3}}+b_{2} b_{3} \mathrm{e}^{\theta_{2}+\theta_{3}}+b_{1} b_{2} b_{3} \mathrm{e}^{\theta_{1}+\theta_{2}+\theta_{3}-\Delta_{13}}
\end{aligned}
$$

Then

$$
u=\left\{\begin{aligned}
& u^{(3)}+u^{(2)}+u^{(1)}=\left(\log \frac{1+a_{3} \mathrm{e}^{\theta_{3}}}{1+b_{3} \mathrm{e}^{\theta_{3}}}\right)_{x}+\left(\log \frac{1+a_{2} \mathrm{e}^{\theta_{2}}}{1+b_{2} \mathrm{e}^{\theta_{2}}}\right)_{x} \\
&+\left(\log \frac{1+a_{1} \mathrm{e}^{\theta_{1}}-\Delta^{\Delta_{13}}}{1+b_{1} \mathrm{e}^{\theta_{1}}-\Delta_{13}}\right)_{x}, y \rightarrow+\infty \\
& u^{(1+2+3)}=\left(\log \frac{1+a_{1} a_{2} a_{3} \mathrm{e}^{\theta_{1}+\theta_{2}+\theta_{3}-\Delta_{13}}}{1+b_{1} b_{2} b_{3} \mathrm{e}^{\theta_{1}+\theta_{2}+\theta_{3}-\Delta_{13}}}\right)_{x}, y \rightarrow-\infty
\end{aligned}\right.
$$

Case 3. Substituting $A_{12} A_{23} \mathrm{e}^{\theta_{2}} \rightarrow \mathrm{e}^{\theta_{2}}$ into Equation (2.3c), and taking the limit of $A_{12}$ and $A_{23}$, we get

$$
\begin{aligned}
g_{3}=1 & +a_{1} \mathrm{e}^{\theta_{1}}+a_{3} \mathrm{e}^{\theta_{3}}+a_{1} a_{3} \mathrm{e}^{\theta_{1}+\theta_{3}-\Delta_{13}} \\
& +a_{1} a_{2} a_{3} \mathrm{e}^{\theta_{1}+\theta_{2}+\theta_{3}-\Delta_{13}} \\
f_{3}= & 1+b_{1} \mathrm{e}^{\theta_{1}}+b_{3} \mathrm{e}^{\theta_{3}}++b_{1} b_{3} \mathrm{e}^{\theta_{1}+\theta_{3}-\Delta_{13}} \\
& +b_{1} b_{2} b_{3} \mathrm{e}^{\theta_{1}+\theta_{2}+\theta_{3}-\Delta_{13}}
\end{aligned}
$$

Then

$$
u=\left\{\begin{aligned}
u^{(3)}+u^{(1+2)}= & \left(\log \frac{1+a_{3} \mathrm{e}^{\theta_{3}}}{1+b_{3} \mathrm{e}^{\theta_{3}}}\right)_{x} \\
& +\left(\log \frac{1+a_{1} a_{2} \mathrm{e}^{\theta_{1}+\theta_{2}-\Delta_{13}}}{1+b_{1} b_{2} \mathrm{e}^{\theta_{1}+\theta_{2}-\Delta_{13}}}\right)_{x}, \quad y \rightarrow+\infty \\
u^{(1)}+u^{(2+3)}= & \left(\log \frac{1+a_{1} \mathrm{e}^{\theta_{1}}}{1+b_{1} \mathrm{e}^{\theta_{1}}}\right)_{x} \\
& +\left(\log \frac{1+a_{2} a_{3} \mathrm{e}^{\theta_{2}+\theta_{3}-\Delta_{13}}}{1+b_{2} b_{3} \mathrm{e}^{\theta_{2}+\theta_{3}-\Delta_{13}}}\right), \quad y \rightarrow-\infty
\end{aligned}\right.
$$

\subsubsection{Minus 2-Resonance}

In the limit of $A_{12} \rightarrow+0, A_{23} \rightarrow+0$, Equation (2.3c) can be rewritten as

$$
\begin{aligned}
& g_{3}=1+a_{1} \mathrm{e}^{\theta_{1}}+a_{2} \mathrm{e}^{\theta_{2}}+a_{3} \mathrm{e}^{\theta_{3}}+a_{1} a_{3} \mathrm{e}^{\theta_{1}+\theta_{3}-\Delta_{13}} \\
& f_{3}=1+b_{1} \mathrm{e}^{\theta_{1}}+b_{2} \mathrm{e}^{\theta_{2}}+b_{3} \mathrm{e}^{\theta_{3}}+b_{1} b_{3} \mathrm{e}^{\theta_{1}+\theta_{3}-\Delta_{13}},
\end{aligned},
$$

Then

$$
u=\left\{\begin{aligned}
u^{(2-3)}+u^{(1)}= & \left(\log \frac{a_{2} \mathrm{e}^{\theta_{2}}+a_{3} \mathrm{e}^{\theta_{3}}}{b_{2} \mathrm{e}^{\theta_{2}}+b_{3} \mathrm{e}^{\theta_{3}}}\right)_{x} \\
& +\left(\log \frac{1+a_{1} \mathrm{e}^{\theta_{1}-\Delta_{13}}}{1+b_{1} \mathrm{e}^{\theta_{1}-\Delta_{13}}}\right)_{x}, y \rightarrow+\infty \\
u^{(3)}+u^{(1)}= & \left(\log \frac{1+a_{3} \mathrm{e}^{\theta_{3}-\Delta_{13}}}{1+b_{3}^{\theta_{3}-\Delta_{13}}}\right)_{x}, \\
& +\left(\log \frac{1+a_{1} \mathrm{e}^{\theta_{1}}}{1+b_{1} \mathrm{e}^{\theta_{1}}}\right)_{x}, \quad y \rightarrow-\infty
\end{aligned}\right.
$$

The case of condition $\Delta_{12} \rightarrow \pm \infty, \Delta_{13} \rightarrow \pm \infty$ and $\Delta_{13} \rightarrow \pm \infty, \Delta_{23} \rightarrow \pm \infty$ are similar.

By the asymptotic analysis above, we know that two types of 2-resonance 3-soliton solution possess four arms (See Figures 4(b)-(d)), the 2-soliton solution has also four arms, but differently, the behaviors of the former in the intermediate region are not stationary.

\subsection{3-Resonance}

For the plus 3-resonance, substituting $A_{12} \mathrm{e}^{\theta_{1}} \rightarrow \mathrm{e}^{\theta_{1}}$, $A_{23} \mathrm{e}^{\theta_{2}} \rightarrow \mathrm{e}^{\theta_{2}}, A_{13} \mathrm{e}^{\theta_{3}} \rightarrow \mathrm{e}^{\theta_{3}}$ into Equation (2.3c), and taking the limit of $A_{12} \rightarrow \infty, A_{13} \rightarrow \infty, A_{23} \rightarrow \infty$, we get

$$
\begin{aligned}
& g_{3}=1+a_{1} a_{2} a_{3} \mathrm{e}^{\theta_{1}+\theta_{2}+\theta_{3}} \\
& f_{3}=1+b_{1} b_{2} b_{3} \mathrm{e}^{\theta_{1}+\theta_{2}+\theta_{3}}
\end{aligned}
$$

this case is like 1-soliton solution, which only has one arm.

For the minus 3-resonance, by taking the limit 


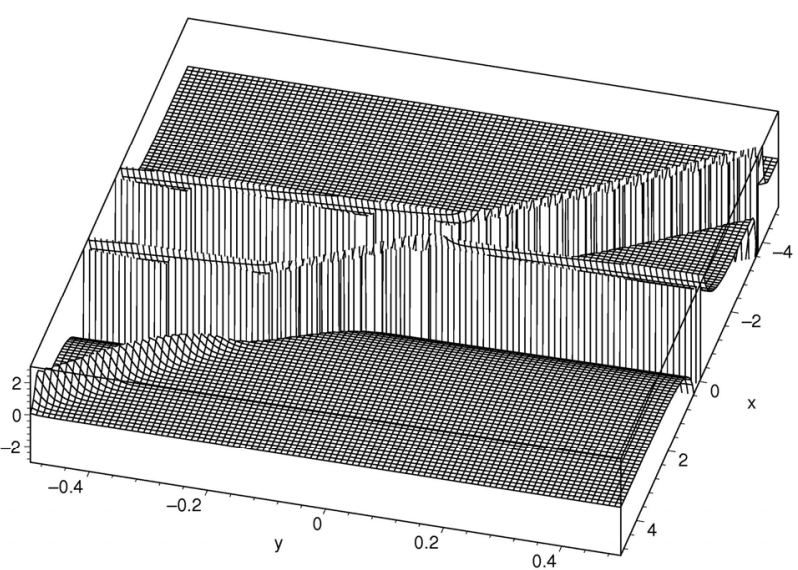

(a)

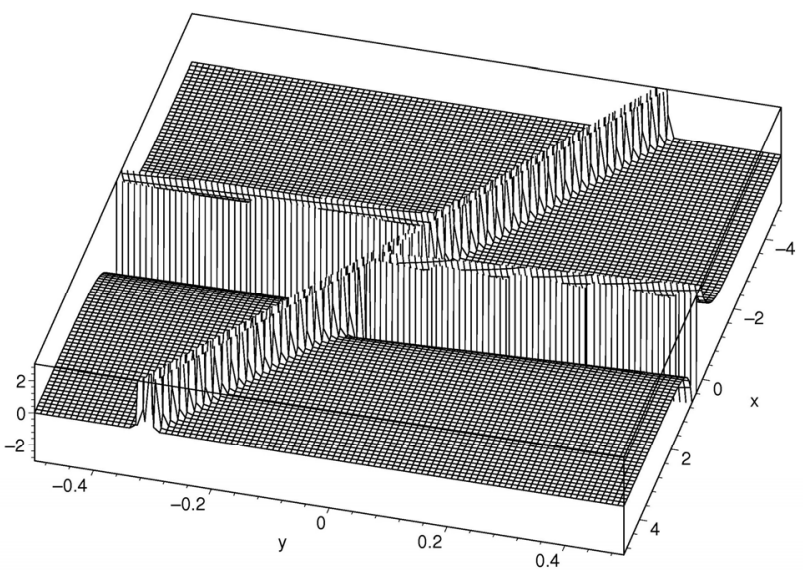

(c)

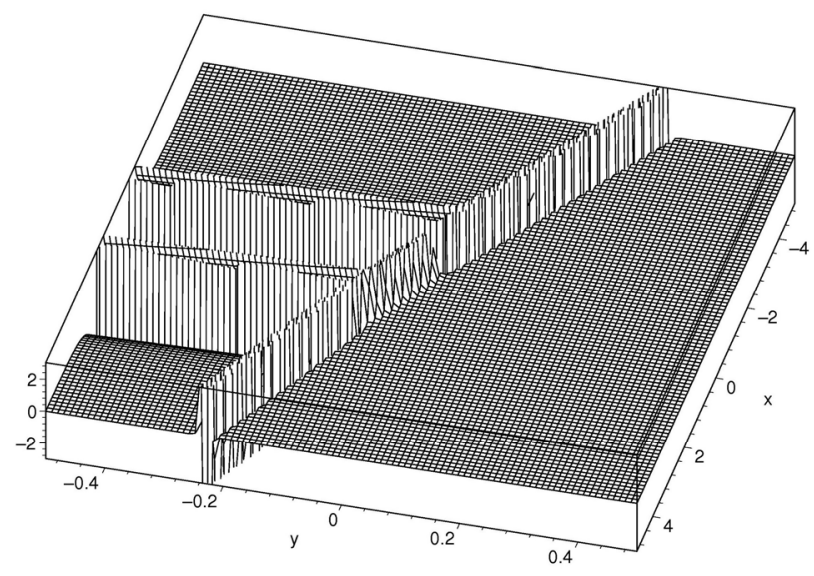

(e)

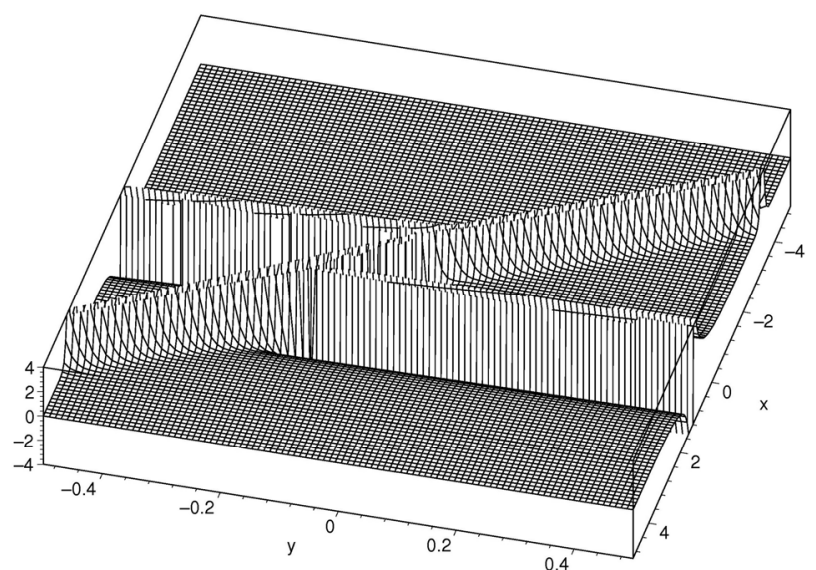

(b)

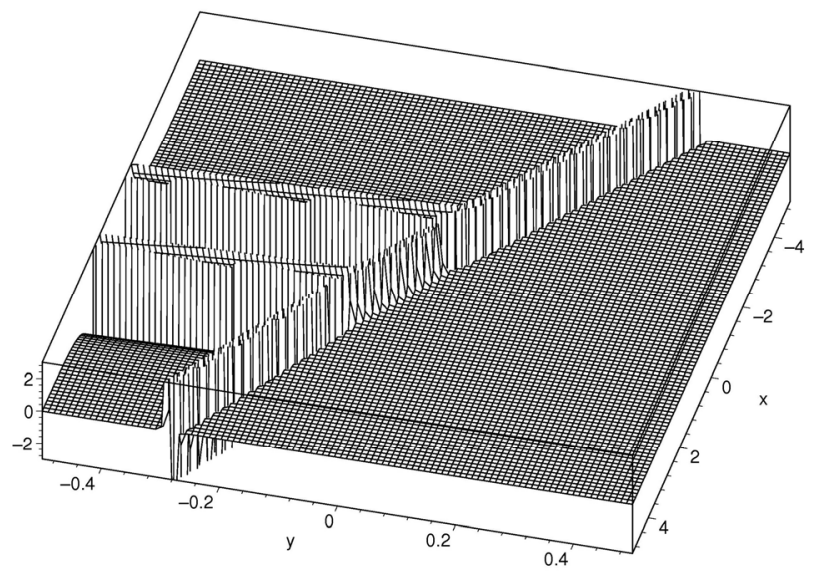

(d)

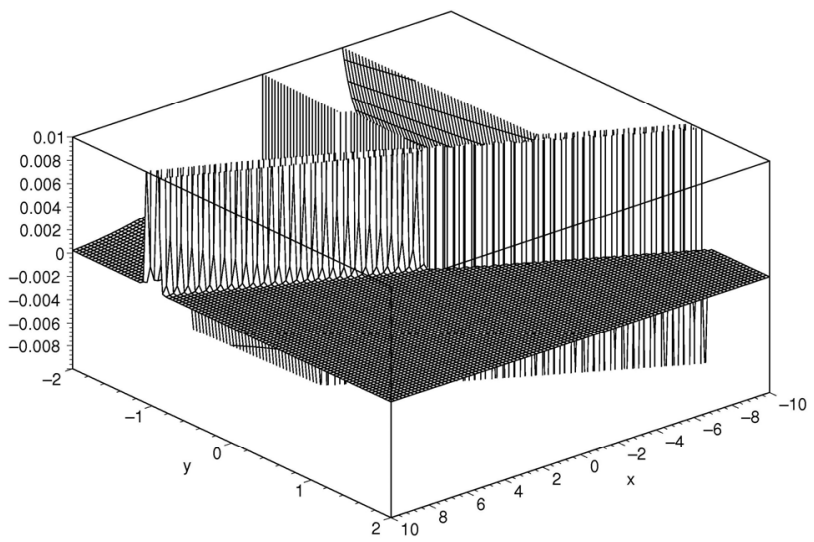

(f)

Figure 4. Minus and plus resonance of 3-soliton solution, (a) 3-soliton minus 1-resonance; (b) 3-soliton plus 2-resonance case 1; (c) 3-soliton plus 2-resonance case 2; (d) 3-soliton plus 2-resonance case 3; (e) 3-soliton minus 2-resonance; (f) 3-soliton minus 3-resonance. 
$A_{12} \rightarrow+0, A_{13} \rightarrow+0, A_{23} \rightarrow+0$ of the Equation (2.3c), we have

$$
\begin{aligned}
& g_{3}=1+a_{1} \mathrm{e}^{\theta_{1}}+a_{2} \mathrm{e}^{\theta_{2}}+a_{3} \mathrm{e}^{\theta_{3}} \\
& f_{3}=1+b_{1} \mathrm{e}^{\theta_{1}}+b_{2} \mathrm{e}^{\theta_{2}}+b_{3} \mathrm{e}^{\theta_{3}}
\end{aligned}
$$

Then

$$
u=\left\{\begin{aligned}
u^{(3)}+u^{(2-3)}= & \left(\log \frac{1+a_{3} \mathrm{e}^{\theta_{3}}}{1+b_{3} \mathrm{e}^{\theta_{3}}}\right)_{x} \\
& +\left(\log \frac{a_{2} \mathrm{e}^{\theta_{2}}+a_{3} \mathrm{e}^{\theta_{3}}}{b_{2} \mathrm{e}^{\theta_{2}}+b_{3} \mathrm{e}^{\theta_{3}}}\right), \quad y \rightarrow+\infty \\
u^{(1-2)}+u^{(1)}= & \left(\log \frac{a_{1} \mathrm{e}^{\theta_{1}}+a_{2} \mathrm{e}^{\theta_{2}}}{b_{1} \mathrm{e}^{\theta_{1}}+b_{2} \mathrm{e}^{\theta_{2}}}\right)_{x} \\
& +\left(\log \frac{1+a_{1} \mathrm{e}^{\theta_{1}}}{1+b_{1} \mathrm{e}^{\theta_{1}}}\right)_{x}, \quad y \rightarrow-\infty,
\end{aligned}\right.
$$

which has four arms (See Figure 4(f)).

\section{Conclusions}

In this work, we have primarily focused on the asymptotic behavior of the $\$ 2 \$$ - and $\$ 3 \$$-soliton solution as $x . y \rightarrow \pm \infty$ and their interactions in the $x y$ plane. Generally, in the case of multi-soliton, saying N-soliton solutions, it has 1-, 2-, $\cdots, C_{N}^{2}$ - resonance N-soliton solutions, and all of them have minus and plus ones. The condition will be more complicated with the increase of $N$. A full characterization of interaction patterns of the general ones is an important open problem, which is left for further study. It is pointed out that the amplification of the amplitude has been experimentally observed and has practical in maritime security and coastal engineering. It has been found out that many soliton equations have resonance phenomenon which will be helpful in making further investigation on the interaction and energy distribution of gravity waves, and evaluating the impact on the ship traffic on the surface of water. We expect that the results presented in this work will be useful to study solitonic solutions in a variety of integrable systems.

\section{Acknowledgements}

This work is supported by the National Natural Science Foundation of China (No 10831003), the Natural Science Foundation of Zhejiang Province (No Y7080198 and No R6090109).

\section{References}

[1] M. J. Ablowitz and P. A. Clarkson, "Solitons, Nonlinear Evolution Equations and Inverse Scattering,” Cambridge
University Press, Cambridge, 1991. doi:10.1017/CBO9780511623998

[2] V. B. Matveev and M. A. Salle, "Darboux Transformation and Solitons," Springer-Verlag, Berlin, Heidelberg, 1991.

[3] R. Hirota, "Exact Solution of the Korteweg-de Vries Equation for Multiple Collisions of Solitons,” Physical Review Letters, Vol. 27, No. 18, November 1971, pp. 1192-1194. doi:10.1103/PhysRevLett.27.1192

[4] R. Hirota, “The Direct Method in Soliton Theory,” Cambridge University Press, Cambridge, 2004.

[5] R. Hirota and J. Satsuma, "Nonlinear Evolution Equations Generated from the Bäcklund Transformation for the Boussinesq Equation,” Progress of Theoretical Physics, Vol. 57, No. 3, September 1977, pp. 797-807. doi:10.1143/PTP.57.797

[6] J. W. Miles, "Resonantly Interacting Solitary Waves," Journal of Fluid Mechanics, Vol. 79, No. 1, April 1977, pp. 171-179. doi:10.1017/S0022112077000093

[7] R. Hirota and M. Ito, "Resonance of Solitons in One Dimension," Journal of the Physical Society of Japan, Vol. 52, No. 3, August 1983, pp.744-748. doi:10.1143/JPSJ.52.744

[8] M. Musette, F. Lambert and J. C. Decuyper, "Soliton and Antisoliton Resonant Interactions,” Journal of Physics A: Mathematical and General, Vol. 20, No. 18, December 1987, pp. 2207-2208. doi:10.1088/0305-4470/20/18/022

[9] F. Lambert, M. Musette and E. Kesteloot, "Soliton Resonances for the Good Boussinesq Equation,” Inverse Problem, Vol. 3, No. 3, May 1987, pp. 275-288. doi:10.1088/0266-5611/3/2/010

[10] K. Ohkuma and M. Wadati, “The Kadomtsev-Petviashvili Equation: The Trace Method and the Soliton Resonances," Journal of the Physical Society of Japan, Vol. 52, No. 3, September 1983, pp. 749-760. doi:10.1143/JPSJ.52.749

[11] E. Medina, “An \$N\$ Soliton Resonance Solution for the KP Equation: Interaction with Change of Form and Velocity," Letters in Mathematical Physics, Vol. 62, No. 2, 2002, pp. 91-99. doi:10.1023/A:1021647025621

[12] O. K. Pashaev and M. L. Y. Francisco, "Degenerate FourVirtual-Soliton Resonance for the KP-II Equation,” Theoretical and Mathematical Physics, Vol. 144, No. 1, 2005 , pp. 1022-1029. doi:10.1007/s11232-005-0130-X

[13] G. Biondini and S. Chakravarty, "Soliton Solutions of the Kadomtsev-Petviashvili II Equation,” Journal of Mathematical Physics, Vol. 47, No. 3, February 2006, pp. 1-26. doi:10.1063/1.2181907

[14] S. Isojima, R. Willox and J. Satsuma, "On Various Solutions of the Coupled KP Equation,” Journal of Physics A: Mathematical and General, Vol. 35, No. 32, May 2002, pp. 6893-6909. doi:10.1088/0305-4470/35/32/309

[15] S. Isojima, R. Willox and J. Satsuma, "Spider-Web Solutions of the Coupled KP Equation,” Journal of Physics A: Mathematical and General, Vol. 36, No. 36, June 2003, pp. 9533-9552. doi:10.1088/0305-4470/36/36/307 
[16] J. H. Lee, R. Willox and O. K. Pashaev, "Soliton Resonances for the MKP-II," Theoretical and Mathematical Physics, Vol. 144, No. 1, July 2005, pp. 995-1003. doi:10.1007/s11232-005-0127-5

[17] H. H. Hao and D. J. Zhang, "Resonances of Line Solitons in a Non-Isospectral Kadomtsev-Petviashvili Equation," Journal of the Physical Society of Japan, Vol. 77, April 2008, Paper ID: 045001. doi:10.1143/JPSJ.77.045001

[18] L. M. Alonso, E. L. Medina and R. Hernandez, "Multidimensional Localized Coherent Structures in the Bilinear Formalism of Integrable Systems,” Inverse Problem, Vol. 7, No. 3, June 1991, p. L25. doi:10.1088/0266-5611/7/3/001

[19] F. Kaka and N. Yajima, "Interaction of Ion-Acoustic Solitons in Two-Dimensional Space," Physical Society of Japan, Vol. 49, November 1980, pp. 2063-2071.
doi:10.1143/JPSJ.49.2063

[20] F. Kaka and N. Yajima, "Interaction of Ion-Acoustic Solitons in Multi-Dimensional Space II,” Physical Society of Japan, Vol. 51, January 1982, pp. 311-322. doi:10.1143/JPSJ.51.311

[21] Y. Zhang, S. F. Deng, D. J. Zhang and D. Y. Chen, “The N-Soliton Solutions for the Non-Isospectral Mkdv Equation,” Vol. 339, No. 3-4, August 2004, pp. 228-236.

[22] S. F. Deng, "The Multisoliton Solutions for the Nonisospectral mKP Equation,” Physics Letters A, Vol. 372, No. 4, January 2008, pp. 460-464. doi:10.1016/j.physleta.2007.07.060

[23] Y. Zhang and Y. N. Lv, "On the Nonisospectral Modified Kadomtsev-Peviashvili Equation,” Journal of Mathematical Analysis and Applications, Vol. 342, No. 1, June 2008, pp. 534-541. doi:10.1016/j.jmaa.2007.12.032 